\title{
Focal neuropathy
}

National Diabetes Information Clearinghouse (NDIC)

\section{Definitions}

Neuropathy

Defined by National Diabetes Information Clearinghouse (NDIC)

\section{Source}

National Diabetes Information Clearinghouse (U.S.). (2009). The diabetes dictionary. [Bethesda, Md.]: U.S. Dept. of Health and Human Services, National Institutes of Health, National Institute of Diabetes and Digestive and Kidney Diseases, National Diabetes Information Clearinghouse.

A type of neuropathy in which a single nerve or a group of nerves is affected, producing sudden weakness or pain. 